\title{
Magnetic order of FeMn alloy on the W(001) surface
}

\author{
Martin Ondráček, Josef Kudrnovský, and František Máca * \\ a Institute of Physics ASCR, Na Slovance 2, CZ-182 21 Prague 8, Czech Republic
}

\begin{abstract}
We investigate theoretically the ground state of the FeMn binary alloy monolayer on the W(001) surface, the stability of different magnetic configurations (ferro/antiferromagnetic, disordered local moments, etc.) and estimate concentrations at which a transition occurs between different magnetic orders. The tight-binding linear muffin-tin orbital method combined with the coherent potential approximation is used to treat the surface alloy appropriately. We discuss the role of disorder on the phase transitions in surface alloys composed from two different $3 d$ transition metals.
\end{abstract}

Key words: Manganese; Iron; Alloy; Surface magnetism; Density functional calculations PACS: 75.70.Ak, 71.15.Nc, 71.20.Be

\section{Introduction}

Magnetic phenomena at surfaces and interfaces have been studied extensively in recent years. This interest in surface magnetism has been stimulated by the needs of technology (magnetic storage media, spintronics) as well as the development of tools for research in this field, both experimental (e.g. the spin-polarized scanning tunneling microscope) [1] and computational (increasing speed and memory capacity of computers).

Reduced number of nearest neighbors at surfaces and lowering of crystal symmetry due to the presence of the surface are known to have a deep impact on magnetic properties. The magnetic moments of atoms in the surface layer are often substantially enhanced as compared to corresponding

\footnotetext{
* Corresponding author.

Email address: maca@fu.cz (František Máca).
}

local magnetic moments inside the bulk. Elements that are known to be nonmagnetic in their bulk form can be magnetic when placed on a surface. An interesting example of how the chemical and geometrical properties of a surface may influence the magnetism of an adsorbate has been reported recently. Experiments indicated that iron monolayers on the (001) tungsten surface had zero net magnetization (see [2] and references therein), in contrast, e.g., to iron monolayers on the W(110) surface that are known to be ferromagnetic [3]. The question whether they were really non-magnetic or rather antiferromagnetic was resolved by Kubetzka [4] using the spin-polarized scanning tunneling microscope. Here, antiferromagnetic $c(2 \times 2) \mathrm{Fe}-$ monolayer on the $\mathrm{W}(001)$ was directly observed. The subsequent theoretical study by Ferriani [5] showed that iron and cobalt monolayers tend to be antiferromagnetic on the $\mathrm{W}(001)$ surface while $\mathrm{V}$, $\mathrm{Cr}$ and $\mathrm{Mn}$ monolayers tend to the ferromagnetic 
order. It should be noted that just an opposite trend is observed on the W(110) surface, on which $\mathrm{V}, \mathrm{Cr}$, and Mn monolayers are $c(2 \times 2)$ antiferromagnets while Fe and Co order ferromagnetically. The same or similar situation as on the W(110) surface occurs also on many other substrates, e.g. on $\mathrm{Cu}, \mathrm{Ag}$ and $\mathrm{Pd}$ (001) surfaces (see references in $[5])$.

Our goal is to generalize the above study to the case of binary two-dimensional alloys on the $\mathrm{W}(001)$ surface. The disorder in the surface layer and the interaction between chemically different surface atoms are expected to lead to new types of magnetic phases. We have focused on the FeMn system in which alloy constituents exhibits antiferromagnetic ( $\mathrm{Fe})$ and ferromagnetic $(\mathrm{Mn})$ order as pure overlayers. The frustration that results from the competing interactions will play a key role in formation of more complex magnetic structures.

\section{Method}

The electronic structure was described in the framework of the tight-binding linear muffin-tin orbital (TB-LMTO) method with the atomic sphere approximation (ASA) [6]. The spin-polarized local density approximation (LDA) with the parametrization of Vosko, Wilk, and Nusair [7] for the exchange-correlation potential was used. The spin-orbit interaction was neglected. The surface Green function formalism was used to describe the electronic structure of an overlayer on substrate with realistic boundary conditions. The substitutional disorder was treated by means of the coherent potential approximation (CPA), which neglects local environment effects. In addition to the standard ASA, a dipole surface barrier was included.

The geometry of the system is the body-centered cubic (bcc) lattice of tungsten with the experimental lattice constant. The self-consistent electronic structure calculations were performed on an eight-layer slab: the slab consisted of four tungsten layers, one layer of $3 d$ transition-metal alloy, and three empty layers that mimicked the vacuum above the surface. The slab was then matched to a semi-infinite vacuum region on one side and to a semi-infinite tungsten bulk on the other side. The layers of the surface slab form an ideal continuation of the bulk structure and possible layer relaxations are neglected.

We have tested the stability of various magnetic phases of two-dimensional random overlayers on the $\mathrm{W}(001)$ substrate. In addition to the ferromagnetic (FM) phase, we have also considered the DLM phase (disordered local moments), the so-called APM phase (antiparallel arrangement of moments), and the uncompensated DLM (UDLM) phase.

The DLM phase is defined by randomly oriented local magnetic moments. The distribution of directions is uniform in the whole space. Such a structure can be efficiently studied using the spinpolarized CPA: the infinite number of possible orientations of local moments can be replaced by two equally large groups of atoms with opposite orientations of local moments. The DLM phase of a binary alloy $\mathrm{X}_{c} \mathrm{Y}_{1-c}$ can be formally described as a four-component alloy $\mathrm{X}_{c / 2}^{+} \mathrm{X}_{c / 2}^{-} \mathrm{Y}_{(1-c) / 2}^{+} \mathrm{Y}_{(1-c) / 2}^{-}$, where the superscripts denote the orientations of respective local moments. The DLM structure has a zero total magnetization similarly as any antiferromagnetic structure. In contrast to an ordered antiferromagnetic structure, however, the local moments in the DLM phase are distributed on the lattice randomly. Energetic preference of the DLM arrangement to the ferromagnetic one is an indication that the antiferromagnetic arrangement would be favored over ferromagnetism.

The APM arrangement in a binary alloy is defined as a magnetic phase in which all local moments of one component are aligned in one direction and all local moments of the other component are aligned in the opposite direction. The APM phase can be described as $\mathrm{X}_{c}^{+} \mathrm{Y}_{1-c}^{-}$. This arrangement resembles a ferrimagnetic one in a similar way as the DLM resembles antiferromagnetism. It should be noted that both the antiferromagnet or ferrimagnet assume the existence of two different sublattices while the DLM or APM arrangement exist on one lattice.

A mixed magnetic state, which we denote as $\operatorname{DLM}(\mathrm{X})+\mathrm{FM}(\mathrm{Y})$, can be represented by a ternary 
compound $\mathrm{X}_{c / 2}^{+} \mathrm{X}_{c / 2}^{-} \mathrm{Y}_{1-c}^{+}$. The most general phase is the uncompensated DLM state (UDLM). It can be approximated as a four-component alloy $\mathrm{X}^{+} \mathrm{X}^{-} \mathrm{Y}^{+} \mathrm{Y}^{-}$where all occupation probabilities (concentrations), $c_{X}^{+}, c_{X}^{-}, c_{Y}^{+}, c_{Y}^{-}$, are arbitrary with the obvious constraints $c_{X}^{+}+c_{X}^{-}=c$ and $c_{Y}^{+}+c_{Y}^{-}=1-c$. All previously discussed phases can be viewed as special cases of the UDLM.

\section{Results and discussion}

The local magnetic moments of FM, DLM and APM phases of the FeMn/W(001) alloy are shown in Fig. 1 and contrasted with behavior of the $\mathrm{FeV} / \mathrm{W}(001)$ system. Magnetic moments of $\mathrm{Mn}$ and also of Fe are rigid, only weakly influenced by their environment.

Total energies for the FeMn/W(001) alloys in different magnetic phases are presented in Fig. 2. To analyze it, let us consider pairwise magnetic interactions only and suppose that the magnetic interaction between two atoms is determined by the atoms themselves independently of their environment. We can then approximate the dependence of energy on magnetic configuration by a simple quadratic form

$$
\begin{aligned}
E-E^{D L M}= & p\left(c_{X}^{+}-c_{X}^{-}\right)^{2}+q\left(c_{Y}^{+}-c_{Y}^{-}\right)^{2} \\
& +2 r\left(c_{X}^{+}-c_{X}^{-}\right)\left(c_{Y}^{+}-c_{Y}^{-}\right) .
\end{aligned}
$$

Specifically, we get:

$$
\begin{aligned}
E^{F M}-E^{D L M} & =p c_{X}^{2}+q c_{Y}^{2}+2 r c_{X} c_{Y} \\
E^{A P M}-E^{D L M} & =p c_{X}^{2}+q c_{Y}^{2}-2 r c_{X} c_{Y} \\
E^{D L M+F M}-E^{D L M} & =q c_{Y}^{2} .
\end{aligned}
$$

The parameters $p, q$, and $r$ have been chosen to fit the calculated total energies of the ferromagnetic $\mathrm{Fe} / \mathrm{W}(001)$ surface, the ferromagnetic $\mathrm{Mn} / \mathrm{W}(001)$ surface, and the $\mathrm{Fe}_{0.5} \mathrm{Mn}_{0.5} / \mathrm{W}(001)$ surface in the APM phase.

As can be seen from Fig. 2, the $\mathrm{DLM}(\mathrm{Fe})+\mathrm{FM}(\mathrm{Mn})$ phase has the lowest energy in the iron-rich FeMn overlayers. The $\operatorname{DLM}(\mathrm{Fe})+\mathrm{FM}(\mathrm{Mn})$ phase allows both elements to retain their preferred magnetic state. In manganese-rich overlayers, the APM

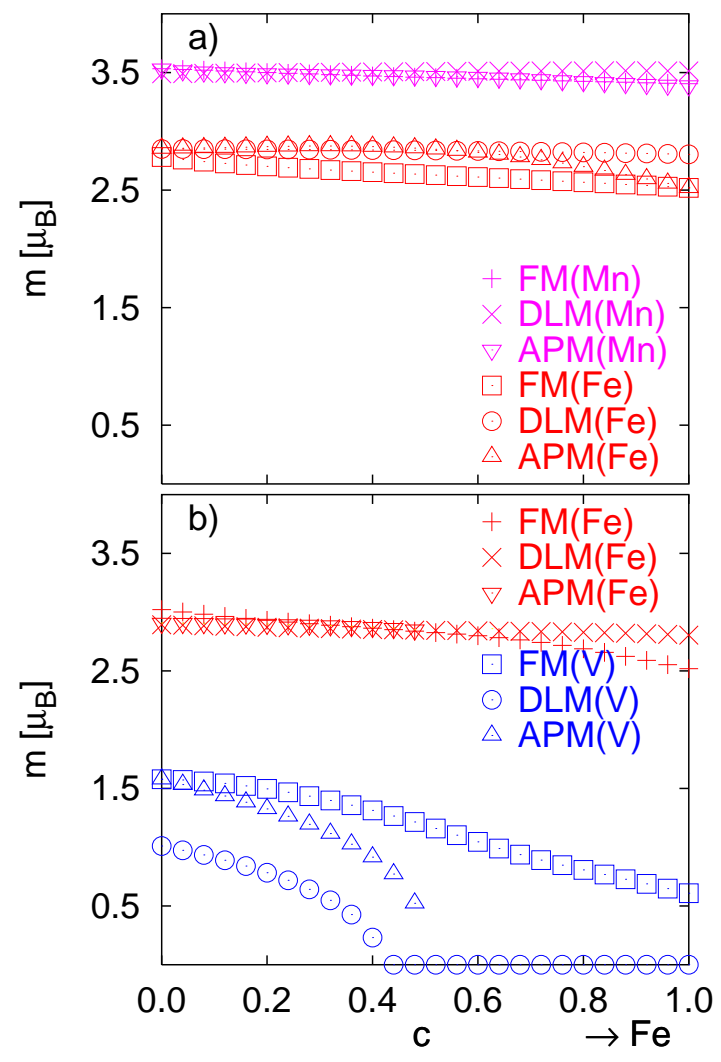

Fig. 1. a) Local magnetic moments at atoms of the FeMn surface alloy as a function of iron concentration in various magnetic states. b) The same for the FeV surface alloy.

phase has lower total energy as compared to the DLM+FM phase. In the APM phase, the Mn local moments keep their parallel (ferromagnetic) arrangement while the Fe local moments are oriented antiparallel with respect to the Mn ones. We can trace three tendencies that drive the magnetic behavior of the $\mathrm{FeMn} / \mathrm{W}(001)$ system: (i) local moments of $\mathrm{Fe}$ atoms tend to be oriented antiparallel to each other, (ii) local moments of Mn atoms tend to align parallel to each other, and (iii) the local moments of Fe tend to be oriented antiparallel to Mn moments.

The parameters in (1) express the strength of pair magnetic interactions between atoms in the overlayer: $p$ describes the interactions between $\mathrm{Fe}$ atoms, $q$ between Mn atoms, and $r$ between Fe and $\mathrm{Mn}$ atoms. The qualitative agreement between this model and the self-consistently calculated results 


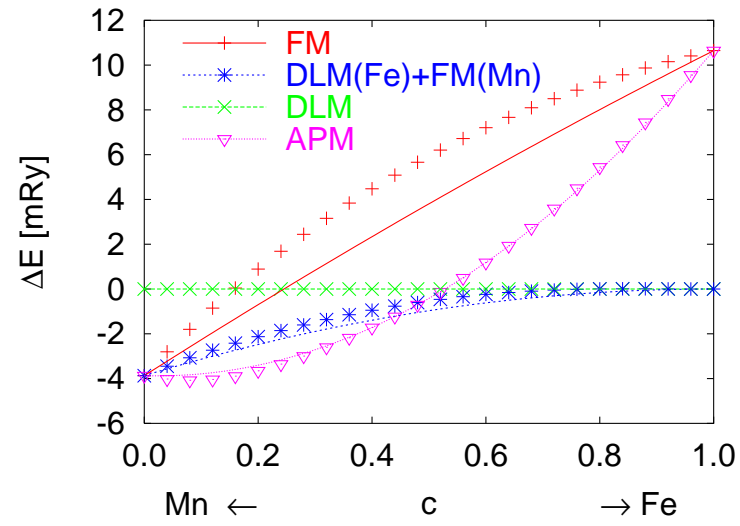

Fig. 2. Total energies of various magnetic phases of the FeMn surface alloy with respect to the reference DLM state as a function of iron concentration. Symbols denote ab-initio calculated values, lines show values obtained from the model described in the text.

(cf. Fig. 2) justifies our discussion of the results in terms of the three driving tendencies that correspond to these three parameters. Our preliminary studies indicate that this model is applicable to other $3 d$ transition metal alloy overlayers with rigid magnetic moments, but not to, e.g., vanadium alloys where more complex behavior has been found.

\begin{tabular}{|l|c|c|c|c|c|}
\hline composition & $\mathbf{c}_{\mathbf{X}}^{+} / \mathbf{c}_{\mathbf{X}}$ & $\mathbf{c}_{\mathbf{Y}}^{+} / \mathbf{c}_{\mathbf{Y}}$ & $\overline{\mathbf{m}}_{\mathbf{X}}\left[\mu_{\mathbf{B}}\right]$ & $\overline{\mathbf{m}}_{\mathbf{Y}}\left[\mu_{\mathbf{B}}\right]$ & state \\
\hline $\mathrm{Fe}_{0.25} \mathrm{Mn}_{0.75}$ & 1.00 & 0.00 & 2.87 & 3.49 & $\mathrm{APM}$ \\
$\mathrm{Fe}_{0.50} \mathrm{Mn}_{0.50}$ & 0.75 & 0.00 & 2.85 & 3.49 & $\mathrm{UDLM}$ \\
$\mathrm{Fe}_{0.75} \mathrm{Mn}_{0.25}$ & 0.60 & 0.00 & 2.83 & 3.50 & $\mathrm{UDLM}$ \\
\hline
\end{tabular}

Table 1

UDLM states with the lowest total energy for FeMn surface binary alloys. First column gives the chemical composition. Then the magnetic arrangement with the lowest energy is described; $\bar{m}_{X}\left[\mu_{B}\right]$ and $\bar{m}_{Y}\left[\mu_{B}\right]$ are average local moments of corresponding elements. The abbreviation in the last column characterizes the magnetic state qualitatively.

We have also investigated the UDLM states to find the state with the lowest total energy. Good sampling of possible UDLM configurations requires a large number of calculations. We have therefore performed calculations for only three different compositions of FeMn (see Table 1). It should be noted that for all concentrations Mn moments prefer ferromagnetic alignment $\left(c_{Y}^{+} / c_{Y}=0\right)$.
For some surface alloy compositions, there are UDLM states that have lower total energy than the special magnetic arrangements discussed so far. We have verified that the formula (1) describes fully self-consistent calculations for FeMn alloys with a good accuracy even in the UDLM phase under the assumption that parameters $p, q$, and $r$ are determined from calculated total energies of the DLM, FM, APM, and DLM+FM phases. The existence of UDLM phases is a consequence of the interplay between the three basic trends discussed above.

For pure $\mathrm{Fe}$ and $\mathrm{Mn}$ monolayer is the first interlayer distance reduced by $13.7 \%$ and $4.7 \%$, respectively [5]. To judge the impact of interlayer relaxation on our results, we have reduced distances between FeMn alloy addlayer and the first tungsten layer by $10 \%$. This relaxation has no remarkable influence on magnetic behavior of FeMn alloy film. Nevertheless, the surface relaxation suppress magnetic moments of iron and manganese atoms slightly because of $d$-band broadening due to stronger bonding with substrate.

\section{Conclusions}

We have investigated magnetic ground states of two-dimensional binary FeMn transition-metal alloys on W(001). For pure monolayers we have found that $\mathrm{Fe}$ overlayer order antiferromagnetically while Mn atoms prefer the ferromagnetic alignment. In alloy monolayers, Fe and Mn tend to orient their local magnetic moments antiparallel to each other.

Incompatible tendencies of magnetic moment alignments among $\mathrm{Fe}$ and $\mathrm{Mn}$ atoms lead to an uncompensated DLM phase, which becomes the calculated ground state for some alloy compositions. This indicates tendency to a non-collinear ground state. To reach a more detailed understanding of the alloyed monolayers on W(001), further SP-STM measurements as well as theoretical calculations are needed.

\section{Acknowledgments}

This work has been done within the project AVOZ1-010-0520 of the ASCR. We acknowledge 
the support from the Grant Agency of the Czech

Republic, Contract No. 202/04/583, and the COST P19-OC150 project.

\section{References}

[1] M. Bode, Rep. Prog. Phys. 66 (2003) 523.

[2] W. Wulfhekel et al., Phys. Rev. B 68 (2003) 144416.

[3] H. J. Elmers, and U. Gradmann, Applied Physics A 51 (1990) 255.

[4] A. Kubetzka et al., Phys. Rev. Lett. 94 (2005) 087204.

[5] P. Ferriani, P., S. Heinze, G. Bihlmayer and S. Blügel, Phys. Rev. B 72 (2005) 024452.

[6] I. Turek, V. Drchal, J. Kudrnovský, M. Šob, and P. Weinberger, Electronic Structure of Disordered Alloys, Surfaces and Interfaces (Kluwer Academic Publishers, Boston-London-Dordrecht, 1997).

[7] S. H. Vosko, L. Wilk, and M. Nusair, Can. J. Phys. 58 (1980) 1200. 